\title{
Temperature level optical fiber sensor using shape memory alloy wires
}

\author{
J.M. López-Higuera, L. Rodriguez-Cobo, A. Quintela, R. Hidalgo-Gato and H. Shokry \\ Photonic Engineering Group of the University of Cantabria \\ R\&D\&i Telecommunication Building \\ Avda. Los Castros s/n - c.p. 39005 Santander, Spain \\ Tel: + 34-942-201498; Fax: + 34-942-200877; e-mail: lopezhjm@unican.es
}

\begin{abstract}
A temperature Level fiber Optic transducer based on a fiber grating-shape Memory Alloy wire structure (LOMA) is presented in this paper. The basic sensors system works as an on/off optical device: only output light is offered by the device when the structure temperature (T) is over a given temperature level $\left(T>T_{L}\right)$. The proposed fiber structure can be used as point or as quasi-distributed sensors systems exploiting its optical multiplexing capacities. The simple and cost effective device is successfully demonstrated in the laboratory.
\end{abstract}

Keywords: Fiber Bragg Grating sensor, Shape Memory Alloy Wire, Temperature sensor

\section{INTRODUCTION}

Fiber Bragg Grating (FBG) technology have been widely investigated as basic structure of fiber telecommunication devices [1], lasers [2], as the basic structure of optical sensors [3, 4, 5] and sensor networks [6] in a large set of sector applications. Bragg Grating technology can be considered, today, a mature one.

However, the development and availability of new materials enables the conception of new fiber devices using their new technical properties in conjunction with some current fiber optic structures.

In this work, both the Shape Memory Alloy (SMA) wire and the FBG technologies are properly combined to take the advantages of both of them and to enable the creation of a new on/off optical fiber device able to be used in sensing field. The LOMA fiber transducer can be used as a Measurand Level $\left(\mathrm{M}_{\mathrm{L}}\right)$ detector (i.e. to detect a maximum temperature, $\mathrm{T}_{\mathrm{L}}$, or a maximum current, $\mathrm{I}_{\mathrm{L}}$, values). Both, point or quasi-distributed fiber sensors systems can be building-up for a wide set of industrial applications. In this paper, after a brief description of the principle in which this device is based, the experimental setup to demonstrate the concept in the laboratory, the obtained results, their discussion and finally, the extracted conclusions are briefly presented.

\section{PRINCIPLE}

Just to illustrate the proposed transducer concept, a scheme of the device is depicted in the Figure 1a. In short, the LOMA transducer structure is constituted by a Fiber Bragg Grating (FBG) properly fixed to a SMA preloaded with a Bias Force $\left(F_{B}\right)$. A view of the central mentioned part of the structure used in the next section can be observed in the photo 1b. In this case the SMA wire (black) is glued with an special glue to the FBG (epoxied egged in red color).

The Bias Force (given in this particular case with a mini-spring) provokes on the SMA wire length, $l$, a wire elongation $\Delta l$. When the transducer temperature is over a given temperature level $\left(T_{L}\right)$ the wire recovers its original length producing a contraction on the FBG structure length $(\Delta l)$ and, hence a substantial reduction on its Bragg wavelength spectral response due to the applied strain $(\Delta l / l)$.

OFS2012 22nd International Conference on Optical Fiber Sensors, edited by

Yanbiao Liao, Wei Jin, David D. Sampson, Ryozo Yamauchi, Youngjoo Chung, Kentaro Nakamura, Yunjiang Rao,

Proc. of SPIE Vol. 8421, 84214P - (c) 2012 SPIE · CCC code: 0277-786/12/\$18 - doi: 10.1117/12.974952

Proc. of SPIE Vol. 842184214 P-1 
a)

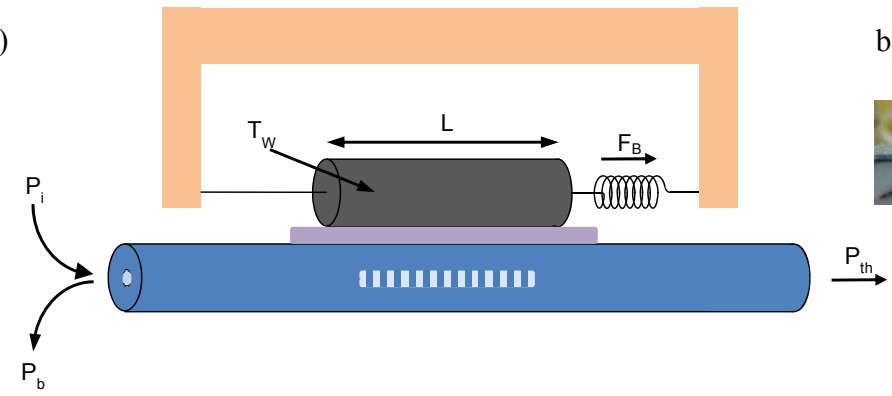

b)

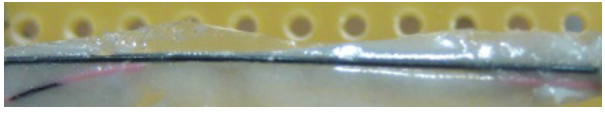

Figure 1. LOMA transducer concept illustration: a) Schematic; b) Photo showing the SMA wire- Fiber Bragg Grating interface.

If the LOMA fiber structure is optically interrogated as illustrated in Figure 2, then an on/off optical sensor device can be constructed. When the measurand (temperature for this paper) level, $T_{L}$, is overpassed, some optical power $\left(\mathrm{P}_{\mathrm{b}}\right)$ is offered by the output sensor port (On) and when the temperature is below the mentioned level (threshold) no optical power is offered by the output sensor port (Off). If the LOMA structure is cooled to the original temperature then the SMA wire recover its original length, $l$, and its original FBG spectral response $\left(\lambda_{\text {LOMA }}\right)$ is also recovered too.

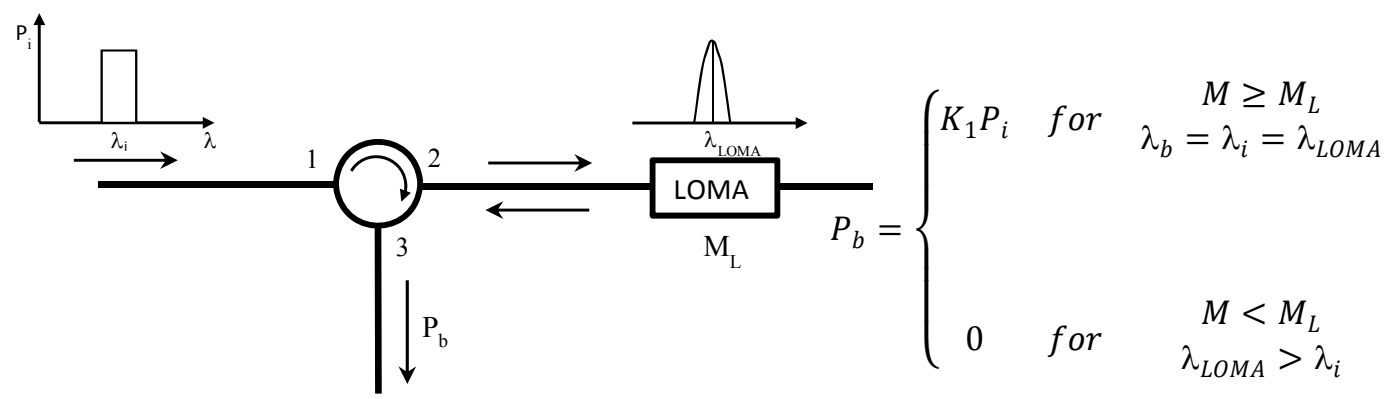

Figure 2. Basic fiber sensor concept architecture. $M=$ measurand; $M_{L}=$ measurand level; $P_{i}$ and $P_{b}$ sensor input and output (back) optical Powers; $\lambda_{i}, \lambda_{L O M A}$ and $\lambda_{b}$ are the input, LOMA and back optical wavelength of the spectra respectively

The SMA actuator wire is based on the shape memory phenomenon which occurs in certain nickel-titanium alloys. When both nickel and titanium atoms are present in the alloy in a 50\%/50\% ratio, the material can forms a crystal structure which is capable of undergoing a change from one crystal form to another (a martensitic transformation) at a temperature determined by the exact composition of the alloy. In the crystal form that exists above the transformation temperature (the austenite) the material is high strength and not easily deformed. It behaves mechanically much like stainless steel. Below the transformation temperature ( $T_{L}$ in this paper), though, when the other crystal form (the martensite) exists, the alloy can be deformed several percent by a very uncommon deformation mechanism that can be reversed when the material is heated and transforms. The low temperature crystal form of the alloy will undergo the reversible deformation fairly easily, so the "memory" strain can be put into the material at rather low stress levels. It is, the SMA wire have the capability to contract by several percent of their length when heated and then easily elongate again by a relatively small load when they are cooled. These materials can be stretched about $4-5 \%$ of its length below its transformation temperature when the biased force $F_{B}$ is about only $69 \mathrm{MPa}$ or less. The transformation temperature can be designed in function of the alloys.

In Figure 3 the temperature elongation cycle of the Flexinol SMA wire used in this paper is shown. 


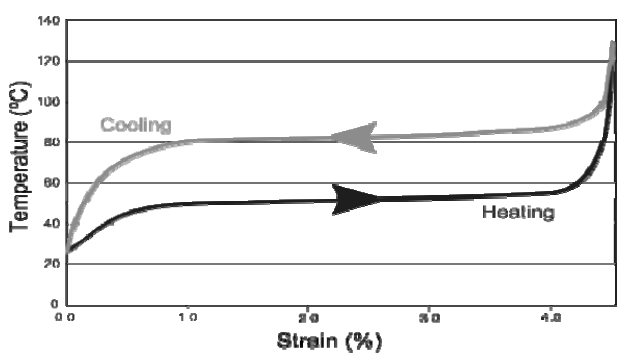

Figure 3. Temperature vs strain behaviors of the SMA wire used in this particular case

\section{EXPERIMENTAL}

The setup used for the experimental demonstration of the proposed transducer concept is depicted in the Figure 4 . The LOMA transducer is heated by the joule effect produced in the SMA wire by the electric current supplied by the HP Controlled Source. The transducer temperature is dynamically monitored and measured using an IRCAN Infrared Thermographic camera equipped with a Macro Lens to be able to "see" the tiny FBG-SMA structure. The LOMA transducer is optically interrogated with a Fibersensing interrogation unit. All equipment's are computer controlled.

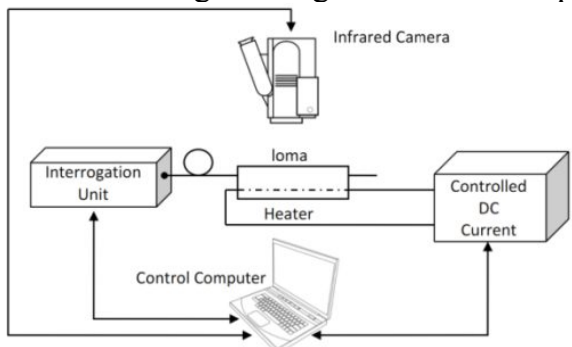

Figure 4. Setup for LOMA concept experimental demonstration

\section{RESULTS AND ITS DISCCUSSION}

The used infrared camera (FLIR ThermaCam SC2000) and a sample of the obtained thermograms sequences can be observed in Figure 5. The spring that supply the Bias Force $F_{B}$ to SMA wire can be deducted on the left of the thermogram.
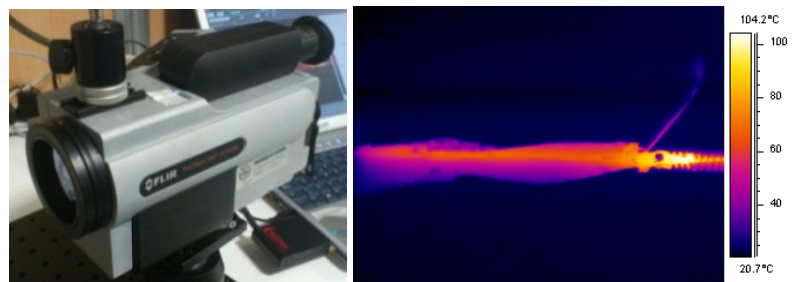

Figure 5. Thermographic camera and one thermogram sample obtained

To check the SMA wire and the fiber transducer behaviours using different current values, the device is heated with nine sequential temperature levels (green colour) followed by a cooling process (no current on the wire). The corresponding spectrum wavelength peaks changes of the transducer response (blue colour) of one of the experiments are summarized in Figure 6a.

In order to check the proposed transducer dynamic device response trains of temperature pulses with temperature peaks over the temperature level $\mathrm{T}_{\mathrm{L}}\left(70^{\circ} \mathrm{C}\right.$ in this case) were applied to the LOMA device. The response of one pulse cycle is shown in the Figure $6 \mathrm{~b}$. 

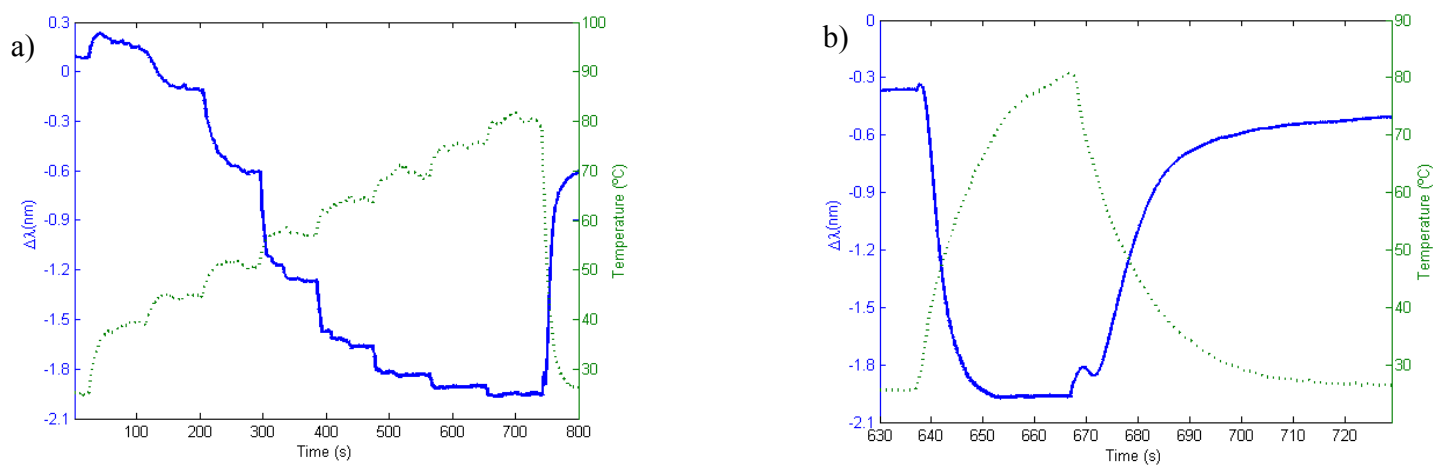

Figure 6. a)LOMA transducer temperature profile measured with the thermographic camera for nine different temperature values following for a cooling process (dotted green line) and the corresponding $\Delta \lambda_{L O M A}$ (solid blue line).b)LOMA wavelength change, $\Delta \lambda_{L O M}$, evolution (solid blue line) for a heat pulse of $80^{\circ} \mathrm{C}$ peak (dotted green line) over the SMA wire temperature level $\left(\mathrm{T}_{\mathrm{L}}=70^{\circ} \mathrm{C}\right)$ of the device

From the obtained results can be deducted that the Bragg wavelength response can by tuneable (in a given range) by temperatures or by electric currents properly controlled and when the temperature device overpass the threshold (level) established by the SMA wire properties then a substantial and saturated wavelength change $\Delta \lambda_{L O M A}$ is obtained. The latter is function of the SMA wire Bias Force $\left(\mathrm{F}_{\mathrm{B}}\right.$ applied in this case for the spring). In this demonstration concept experiment with low $F_{B}$ a change of $1,6 \mathrm{~nm}$ is obtained. It is also demonstrated that finished the heat pulse, the wavelength response is recovered following the cooling process of the LOMA structure and according to the temperature-strain SMA wire behaviours. The sensor level device is also successfully demonstrated in the laboratory according to the principle described in section 2 .

\section{CONCLUSIONS.}

A transducer based on a fiber grating-shape memory alloy wire structure is proposed and experimentally demonstrated in this in this paper. Using this transducer architecture a basic sensors system that works as an on/off optical device, able to work as a temperature level fiber optic sensor, is also reported. The LOMA sensor only offer output light when the structure temperature $(\mathrm{T})$ overpass a given temperature level $\left(T>T_{L}\right)$ or temperature threshold that is established by the SMA wire behaviors. Using a SMA wire with a $70^{\circ} \mathrm{C}$ of transformation temperature (or here $\mathrm{T}_{\mathrm{L}}$ ) both the transducer and the sensor system are experimentally demonstrated in the laboratory. The sensor is successfully checked by a train of thermal pulsed that enables the cooling recovery of the device. This scheme can also be used with other measurands such electric currents.

Thanks to the transducer optical multiplexing properties this device can also be successfully used in fiber optic sensor networks with large number of points or in quasi-distributed sensors as will be shown in further papers in preparation. This simple, cost effective device is patent pending.

\section{ACKNOWLEDGEMENTS}

This work has been supported by the Spanish TEC2010-20224-C02-02 project.

\section{REFERENCES}

[1] Kashyap, Raman 'Fiber Bragg Gratings', Academic Press, ISBN: 0-12-400560-8, 1999.

[2] Cesar Jauregui, Tino Eidam, Jens Limpert, Andreas Tünnermann, "The impact of modal interference on the beam quality of high-power fiber amplifiers". Optics Express, Volume: 19, Issue: 4, Pages: 3258-327, 2011.

[3] J. M. Lopez-Higuera. Handbook of optical fibre sensing technology, Wiley \& Sons (2002).

[4] Culshaw, Brian and Dakin, John, 'Optical Fiber Sensors: vol.I, II, III, and IV' Artech House, Inc.,1996.

[5] Andrea Cusano, Antonello Cutolo and Jacques Albert (Eds)" Fiber Bragg Grating Sensors: Recent Advancements, Industrial Applications and Market Exploitation" Bentham Science Publishers Ltd, 2011.

[6] Manuel López-Amo1 and Jóse Miguel López-Higuera, "Multiplexing Techniques for FBG Sensors", Chapter 6 of the book Fiber Bragg Grating Sensors, Bentham Science Publishers Ltd, 99-115, 2011. 